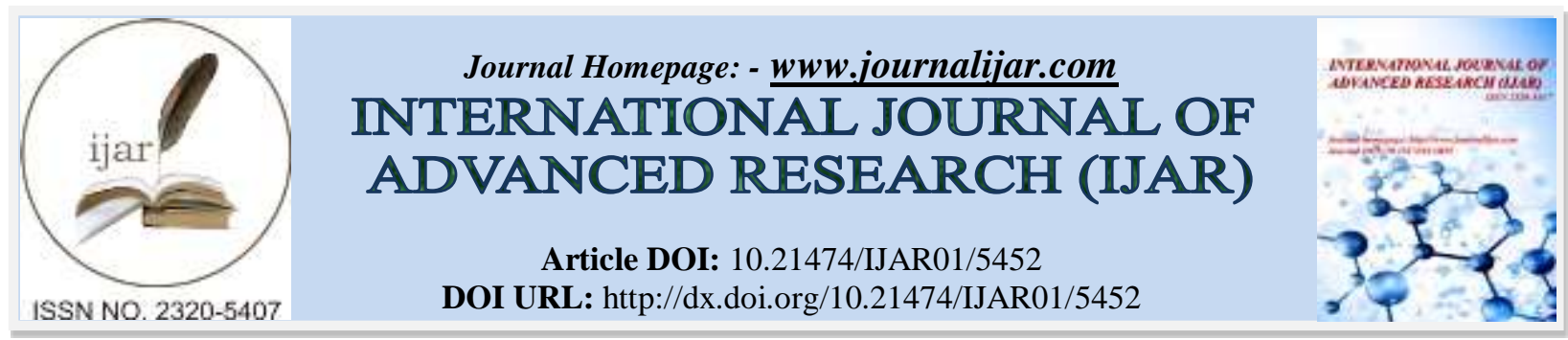

RESEARCH ARTICLE

\title{
ASSESSMENT OF VEGETATION AND PRIORITIZATION OF COMMUNITIES FOR CONSERVATION IN LATAKHARAK ALPINE MEADOWS OF NANDA DEVI BIOSPHERE RESERVE, WEST HIMALAYA, INDIA.
}

\section{S. C. Arya ${ }^{1}$ and S. S. Samant ${ }^{2}$.}

1. G.B. Pant National Institute of Himalayan Environment and Sustainable Development North-East Unit, Vivek Vihar, Itanagar- 791 113, Arunachal Pradesh.

2. G.B. Pant National Institute of Himalayan Environment and Sustainable Development Himachal Unit, MohalKullu-175 126, Himachal Pradesh, India.

\section{Manuscript Info}

\section{Manuscript History}

Received: 17 July 2017

Final Accepted: 19 August 2017

Published: September 2017

Key words:-

Himalaya, Alpine Meadows, Species

Diversity, Nativity, Endemism,

Prioritization

\begin{abstract}
Himalaya is considered as the repository of the biological diversity. The richness of the biological diversity is due to its unique climatic conditions, topography and diverse habitats. The alpine meadows of Himalayan Region are very unique in terms of plant diversity and contains the habitats of many important and high value plant species. They are very rich in native and endemic plant species and very famous its scenic beauty. The present study was carried out to assess the rich plant diversity of alpine meadows in a part of Nanda Devi Biosphere Reserve. The study includes assessment of species richness, species diversity, community diversity, distribution of native, endemic, economically important and rare endangered species within the communities and prioritization of communities for conservation. During the study 10 alpine plant communities supporting 213 species, distributed in 32 sites, 8 habitats, and between $3675-4075 \mathrm{~m}$, amsl. Based on the species richness, native, endemic, economically important and rare endangered species, prioritization of the communities for conservation has been done. Development of adequate strategy and action plan for the conservation of habitats, communities, and ecosystems supporting high value species has been suggested.
\end{abstract}

Copy Right, IJAR, 2017,. All rights reserved.

\section{Introduction:-}

The alpine meadows, locally known as "Bugyal" in Garhwal and Kumaun, Marg in Kashmir and Thach or Dhar in Himachal Pradesh are very unique in terms of topography, climate, plant species. The alpine meadows located above the timber line above the average $3000 \mathrm{~m}$ amsl altitude and consists small herbs, scattered shrubs, mosses and lichens (Billings, 1973; Rau, 1975). The alpine zone occupies nearly 33\% of geographical area in the Himalaya, of which the vegetated and snow-bound areas constitute about $25.88 \%$ and $7.22 \%$, respectively (Anonymous, 1989). These areas are famous for their picturesque beauty, rich floral diversity and repositories of a number of valuable medicinal herbs (Kala et al., 1998). About 2500 plant species have been reported from the alpine zones of the Greater Himalaya of which $c a .200$ are highly endangered, over 100 species are threatened, and 300 species are used for medicinal purposes (Rau, 1975). Due to camping, over grazing and exploitation of medicinal and wild edible plants for

Corresponding Author:- S. C. Arya.

Address:- G.B. Pant National Institute of Himalayan Environment and Sustainable Development

North-East Unit, Vivek Vihar, Itanagar- 791 113, Arunachal Pradesh. 
household use and trade, the habitats of many potential species are degrading fast and over 8 species have been listed in the Red Data Book of Indian Plants (Nayar \& Sastri, 1987, 88, 90; Samant et al., 1996a).

In the alpine region of the Indian Himalaya, a few sporadic studies have been carried out by some workers on community patterns, phenology, biomass and productivity, impact of grazing and prioritization (Kaul \& Sarin 1971; Rawat \& Pangtey, 1987; Pangtey et al., 1990; Sundriyal et al., 1987, 1988; Rawat \& Rodgers, 1988; Joshi et al., 1988; Joshi \& Srivastava 1988, 1991; Ram, 1992; Ram et al., 1988, 1989; Sundriyal, 1989, 1992; Singh, 1991; Singh et al., 1995, Negi et al., 1992, 1993; Rawat \& Uniyal, 1993; Nautiyal et al., 1997; Rikhari et al., 1992; Johnsing et al., 1998; Kala et al., 1998; Kala \& Rawat, 1999; Nautiyal et al., 2001; Raizada et al., 1998; Uniyal et al., 2002; Samant et al., 2002, etc.). However, the vegetation of most of the alpine meadows has not been assessed for sites and habitat characteristics, community diversity (composition and structure), distribution pattern, species diversity, soil composition, distribution pattern of native, endemic, economically important and rare endangered species, and prioritization of communities for conservation. Present attempt has been made on these directions for the first time and integrates these components together to reach some strong conclusions.

\section{The Study Area:-}

Nanda Devi Biosphere Reserve (NDBR) $\left(30^{\circ} 05^{\prime}-31^{\circ} 02^{\prime} \mathrm{N}\right.$ to $\left.79^{\circ} 12^{\prime}-80^{\circ} 19^{\prime} \mathrm{E}\right)$ covering a total of $6,407.03 \mathrm{~km}^{2}$ (Core zone $712.12 \mathrm{~km}^{2}$; Buffer zone $5,148.57 \mathrm{~km}^{2}$ and Transition zone $546.34 \mathrm{~km}^{2}$ ), is situated in the northern part of west Himalaya (Fig. 1) and is among the World Heritage Sites. The reserve includes parts of Bageshwar and Pithoragarh districts in Kumaun region, and Chamoli district in Garhwal region. The buffer and transition zones are inhabited by over 100 villages. Most of the inhabitants belong to two main ethnic groups namely Indo-Mongoloid (Bhotia) and Indo-Aryans. They have been using plants as medicine, edible/food, fodder, fuel, timber, agricultural tools and various other purposes (Samant, 1996b; Joshi et al., 1999, 2001). Present study has been conducted in the Latakharak alpine meadows, located in the Chamoli district, a part of NDBR.

\section{Methods:-}

Identification and Selection of Sites and Habitats:-

Sites have been selected in each and every accessible aspect along transacts between 3000-4270m, amsl. In each site, habitat type, altitude, aspect, slope, boulder percentage and dominant species were noted. Habitats were identified on the basis of physical characters (Samant et al., 1998a). The sites having $>50 \%$ boulders of the ground cover have been identified as bouldery habitat.

\section{Survey, Sampling, Identification and Analysis of Data:-}

The field surveys and samplings were carried out during 1998-2000 within selected sites along transacts. For the sampling of vegetation $20 \times 20 \mathrm{~m}$ plot was marked in each site and 20 quadrats $(1 \times 1 \mathrm{~m})$ in each plot were laid by stratified method. Sampling was done in the peak season i.e., August and September. For data collection and analysis standard ecological methods (Grieg-Smith, 1957; Kersaw, 1973; Muller-Dombois \& Ellenberge, 1974; Dhar et al., 1997) were followed. Shrubs present in each site were noted to update the species composition. From each site, samples of each species were collected and identified in the Institute with the help of florulas and research papers (Naithani, 1984 \& 1985; Polunin \& Stainton, 1984; Samant 1993, 1999; Pangtey et al., 1993; Hajra \& Jain, 1981; and Hajra \& Balodi, 1995). Abundance data of different sites were pooled to get community average in terms of density.

Community Delineation, Identification of Nativity, Endemism, Human Dependence and Rarity:-

Communities have been delineated based on the 50\% contribution of the total relative density of the species. Species of each community have been analyzed for nativity following (Anonymous, 1883-1970; Samant \& Dhar, 1997; Samant, 1999 and Samant et al., 2000), endemism following (Dhar \& Samant, 1993; Samant et al., 1998a\&b, 2000; Samant \& Dhar, 1997; and Samant, 1999), human dependence following (Samant et al. 1996a, Joshi et al., 1999, 2001), rarity following (Samant et al., 1996b, 1998b).

Species Diversity (H'):-

Species diversity was determined by Shanon Wiener's information statistic (H') (Shanon Weiner, 1963). 


\section{Prioritization of Communities for Conservation:-}

Prioritization of the communities for conservation has been done based on the cumulative values of species richness, native, endemic, economically important and rare-endangered species.

\section{Results:-}

Site and Habitat Characteristics:-

Maximum sites (11) have been represented by dry habitat, followed by bouldery and shrubberies ( 4 sites, each), shady moist, rocky and riverine ( 3 sites, each), forest edge ( 2 sites) and camping site (1 site), respectively. Nine sites fall in S aspect, 8 in W aspect, 7 in NW aspect, 4 in N aspect, 2 in SW aspect and, 1 in E and SE aspects, each. The slope varied from $5^{\circ}-65^{\circ}$ and boulder percent from $5-60 \%$ (Table 1 ).

\section{Community Diversity, Distribution Pattern and Species Composition:-}

A total of 10 communities have been delineated in the alpine area falling between $3675-4075 \mathrm{~m}$, amsl. The community types, altitudinal distribution, representation in site/s, habitat/s and major associates have been presented in Table 2. Carex stracheyi represented in maximum sites (16), followed by Danthonia cachemyriana-Carex stracheyi mixed (6 sites), Danthonia cachemyriana (3 sites). Rest of the communities represented in one site only (Table 2).

In general, a total of 227 species (12 shrubs, 215 herbs including 19 pteridophytes) had been recorded. However, 213 species (11 shrubs, 202 herbs including 19 pteridophytes) had been recorded in the sampling sites. Analysis of distribution pattern of species indicated that $81.19 \%$ species had regular distribution pattern; $9.90 \%$ species had random distribution pattern; and $8.91 \%$ species had contagious distribution pattern among the sites; and $85.15 \%$ species had regular distribution pattern; $8.91 \%$ species had random distribution pattern; and $5.94 \%$ species had contagious distribution pattern among the communities.

\section{Community Diversity: Composition and Structure:-}

Species richness among the communities ranged from 6-141. It was highest in Carex stracheyi (141), followed by Danthonia cachemyriana-Carex stracheyi mixed (124), Danthonia cachemyriana (87) and Carex obscura (42), communities (Table 3). The density ranged from 26.95-1767.20 Ind m-2. Carex stracheyi-Danthonia cachemyriana mixed community had maximum density (1767.20 Ind m-2), followed by Danthonia cachemyriana-Carex stracheyi mixed (1687.38 Ind m-2), Danthonia cachemyriana-Cortia depressa-Carex stracheyi mixed (1562.10 Ind m-2) and Danthonia cachemyriana (1004.60 Ind m-2), communities (Table 3).

\section{Species Diversity (H'):-}

Species diversity (H') ranged from 1.39-2.62. It was highest in Fragaria nubicola-Galium acutum-Viola bifloraCardamine impatiens mixed community (2.62), followed by Danthonia cachemyriana-Picrorhiza kurrooa mixed (2.25), Danthonia cachemyriana-Polygonum affine mixed (2.11) and Carex obscura (1.84), communities (Table 3).

\section{Distribution Pattern of the Native and Endemic Species within Communities:-}

Out of the total 227 species, $71.73 \%$ species were natives. Among the natives, $3.70 \%$ species were endemic and 48.77\% species were near endemic and, rest $47.53 \%$ species had wide global distribution. Of the natives, $93.21 \%$ species were herbs and $6.79 \%$ species were shrubs. Endemic species were represented in herbs only whereas near endemic species were represented in both the life forms. Among the near endemic species, $93.67 \%$ species were herbs and $6.33 \%$ species were shrubs.

The distribution of native and endemic species including near endemic ones within the identified communities from Latakharak alpine meadows have been presented in Fig. 2. The maximum natives (99 spp.) were recorded in Carex stracheyi community, followed by Danthonia cachemyriana -Carex stracheyi mixed (89 spp.) and Danthonia cachemyriana (61 spp.), communities. The least natives were recorded in Polygonum polystachyum community (3 spp. only). The maximum endemic species including near endemic ones (52 spp.) were found in Carex stracheyi community, followed by Danthonia cachemyriana-Carex stracheyi mixed (50 spp.) and Danthonia cachemyriana (27 spp.), communities. In Polygonum polystachyum community the endemic and near endemic species were absent. 
Distribution Pattern of the Economically Important Species within Communities:-

From the Latakharak alpine meadows, 125 economically important species $(10$ shrubs, 115 herbs including 2 pteridophytes) belonging to 39 families and 91 genera had been recorded. These species were used for various purposes such as medicine (86 spp.), wild edible/food (38 spp.), fodder (48 spp.), fuel (3 spp.), religious (10 spp.) and various other purposes (11 spp.). Amongst the species, 15 species had multipurpose utility whereas 74 species had single utility.

The richness of economically important species among the identified communities ranged from 3-27. The highest species were distributed in Carex stracheyi (75 spp.), followed by Danthonia cachemyriana-Carex stracheyi (71 spp.), Danthonia cachemyriana (51 spp.) and Carex obscura (27 spp.) communities. The least useful species were distributed in Polygonum polystachyum community (3 spp.).

\section{Distribution Pattern of the Rare Endangered Species within Communities:-}

A total of 38 species ( 1 shrub, 37 herbs including 5 pteridophytes) belonging to 32 genera and 21 families have been identified as threatened from Latakharak alpine meadows. Maximum rare species were distributed in Carex stracheyi community (24 spp.), followed by Danthonia cachemyriana- Carex stracheyi mixed (18 spp.) and Danthonia cachemyriana mixed (10 spp.), communities. The least rare species were distributed in Fragaria nubicola-Galium acutum-Viola biflora-Cardamine impatiens mixed community (1 spp. only). In Polygonum polystachyum community the rare species were absent.

\section{Prioritization of Communities for Conservation:-}

Based on the species richness, native, endemic, economically important and rare endangered species, Carex stracheyi (Total species 141; Natives 99; Endemic 52; Rare-endangered 24; Useful species 75); and Danthonia cachemyriana-Carex stracheyi (Total species 124; Natives 89; Endemic 50; Rare-endangered 18; Useful species 71), communities have been identified as high value communities and prioritized for conservation.

Table 1:- Physical Characteristics of Sites and Habitats.

\begin{tabular}{|l|l|l|l|l|l|l|}
\hline Site & Habitat type & $\begin{array}{l}\text { Altitude } \\
(\mathbf{m})\end{array}$ & Aspect & $\begin{array}{l}\text { Slope } \\
(\mathbf{(})\end{array}$ & $\begin{array}{l}\text { Boulders } \\
\mathbf{\%}\end{array}$ & Dominant species \\
\hline 1. & Camping site & 3715 & $\mathrm{~W}$ & 5 & 5 & $\begin{array}{l}\text { Polygonum polystachyum, Rumex nepalensis, } \\
\text { Fragaria nubicola, Hackelia uncinata }\end{array}$ \\
\hline 2. & Dry & 3715 & $\mathrm{~S}$ & 15 & 5 & $\begin{array}{l}\text { Danthonia cachemyriana, Carex stracheyi, } \\
\text { Trachydium roylei, Carex nubigena }\end{array}$ \\
\hline 3. & Dry & 3700 & $\mathrm{~S}$ & 15 & 5 & $\begin{array}{l}\text { Danthonia cachemyriana, Carex stracheyi, } \\
\text { Dactylis glomerata, Lactuca macrorhiza }\end{array}$ \\
\hline 4. & Dry & 3685 & $\mathrm{E}$ & 20 & 5 & $\begin{array}{l}\text { Danthonia cachemyriana, Carex nubigena, } \\
\text { Carex stracheyi, Eragrostis nigra }\end{array}$ \\
\hline 5. & Forest edge & 3690 & $\mathrm{~N}$ & 25 & 5 & $\begin{array}{l}\text { Danthonia cachemyriana, Anaphalis busua, } \\
\text { Polygonum affine, Carex obscura }\end{array}$ \\
\hline 6. & Dry & 3685 & $\mathrm{~S}$ & 30 & 5 & $\begin{array}{l}\text { Carex stracheyi, Danthonia cachemyriana, } \\
\text { Cortia depressa, Carex obscura }\end{array}$ \\
\hline 7. & Shady moist & 3680 & $\mathrm{~S}$ & 50 & 5 & $\begin{array}{l}\text { Danthonia cachemyriana, Cortia depressa, } \\
\text { Carex stracheyi, Dactylis glomerata }\end{array}$ \\
\hline 8. & Bouldery & 3675 & $\mathrm{~S}$ & 40 & 50 & $\begin{array}{l}\text { Danthonia cachemyriana, Carex stracheyi, } \\
\text { Carex obscura, Cyananthus lobatus }\end{array}$ \\
\hline 9. & Dry & 3720 & $\mathrm{~W}$ & 5 & 5 & $\begin{array}{l}\text { Danthonia cachemyriana, Carex stracheyi, } \\
\text { Cortia depressa, Carex obscura }\end{array}$ \\
\hline 10. & Dry & 3740 & $\mathrm{~W}$ & 25 & 5 & $\begin{array}{l}\text { Carex stracheyi, Danthonia cachemyriana, } \\
\text { Carex setosa, Poa alpina }\end{array}$ \\
\hline 11. & Dry & 3750 & $\mathrm{~S}$ & 45 & 5 & $\begin{array}{l}\text { Carex stracheyi, Cyananthus lobatus, Carex } \\
\text { obscura, Cortia depressa }\end{array}$ \\
\hline 12. & Bouldery & 3750 & $\mathrm{~W}$ & 45 & 50 & $\begin{array}{l}\text { Danthonia cachemyriana, Carex stracheyi, } \\
\text { Carex obscura, Dactylis glomerata }\end{array}$ \\
\hline 13. & Rocky & 3815 & $\mathrm{SW}$ & 65 & 0 & $\begin{array}{l}\text { Carex stracheyi, Danthonia cachemyriana, } \\
\text { Nardostachys grandiflora, Lepisorus tenuipes }\end{array}$ \\
\hline
\end{tabular}




\begin{tabular}{|c|c|c|c|c|c|c|}
\hline 14. & Shady moist & 3850 & SE & 50 & 5 & $\begin{array}{l}\text { Danthonia cachemyriana, Carex stracheyi, } \\
\text { Cyananthus lobatus, Carex nivalis }\end{array}$ \\
\hline 15. & Bouldery & 3850 & $S$ & 25 & 52 & $\begin{array}{l}\text { Danthonia cachemyriana, Carex stracheyi, } \\
\text { Carex setosa, Cyananthus lobatus }\end{array}$ \\
\hline 16. & Riverine & 3875 & $\mathrm{~S}$ & 45 & 25 & $\begin{array}{l}\text { Carex obscura, Dactylis glomerata, Poa } \\
\text { pratensis, Circaea alpina }\end{array}$ \\
\hline 17. & Shrubberries & 3900 & $\mathrm{~N}$ & 25 & 5 & $\begin{array}{l}\text { Carex stracheyi, Viola biflora, Poa pratensis, } \\
\text { Cystopteris fragilis }\end{array}$ \\
\hline 18. & Dry & 3925 & NW & 40 & 5 & $\begin{array}{l}\text { Carex stracheyi, Danthonia cachemyriana, } \\
\text { Cortia depressa, Kobresia duthiei }\end{array}$ \\
\hline 19. & Riverine & 3925 & NW & 35 & 15 & $\begin{array}{l}\text { Carex stracheyi, Danthonia cachemyriana, } \\
\text { Senecio kunthianus, Polygonum affine }\end{array}$ \\
\hline 20. & Shrubberries & 3925 & $\mathrm{~N}$ & 35 & 5 & $\begin{array}{l}\text { Danthonia cachemyriana, Picrorhiza } \\
\text { kurrooa, Polygonum affine, Gentiana kurroo }\end{array}$ \\
\hline 21. & Rocky & 3925 & NW & 65 & 0 & $\begin{array}{l}\text { Carex stracheyi, Carex nubigena, Saxifraga } \\
\text { pulvinaria, Danthonia cachemyriana }\end{array}$ \\
\hline 22. & Shrubberries & 4000 & NW & 50 & 5 & $\begin{array}{l}\text { Carex stracheyi, Polygonum affine, Anaphalis } \\
\text { contorta, Viola biflora }\end{array}$ \\
\hline 23. & Dry & 4000 & W & 50 & 5 & $\begin{array}{l}\text { Carex stracheyi, Danthonia cachemyriana, } \\
\text { Trachydium roylei, Polygonum affine }\end{array}$ \\
\hline 24. & Dry & 4015 & NW & 50 & 5 & $\begin{array}{l}\text { Carex stracheyi, Carex obscura, Polygonum } \\
\text { affine, Carex nubigena }\end{array}$ \\
\hline 25. & Shady moist & 4060 & SW & 40 & 5 & $\begin{array}{l}\text { Carex stracheyi, Danthonia cachemyriana, } \\
\text { Polygonum affine, Nardostachys grandiflora }\end{array}$ \\
\hline 26. & Bouldery & 4075 & $\mathrm{~W}$ & 30 & 60 & $\begin{array}{l}\text { Carex stracheyi, Polygonum affine, Viola } \\
\text { biflora, Anaphalis contorta }\end{array}$ \\
\hline 27. & Shrubberries & 4050 & $\mathrm{NW}$ & 35 & 35 & $\begin{array}{l}\text { Danthonia cachemyriana, Polygonum affine, } \\
\text { Anaphalis contorta, Cerastium cerastioides }\end{array}$ \\
\hline 28. & Riverine & 4050 & $\mathrm{~W}$ & 25 & 10 & $\begin{array}{l}\text { Carex stracheyi, Carex setosa, Thlaspi } \\
\text { andersonii, Picrorhiza kurrooa }\end{array}$ \\
\hline 29. & Rocky & 4060 & $\mathrm{~W}$ & 45 & 0 & $\begin{array}{l}\text { Carex stracheyi, Danthonia cachemyriana, } \\
\text { Nardostachys grandiflora, Polygonum affine }\end{array}$ \\
\hline 30. & Dry & 4010 & $\mathrm{~S}$ & 60 & 5 & $\begin{array}{l}\text { Carex stracheyi, Danthonia cachemyriana, } \\
\text { Trachydium roylei, Saxifraga parnassifolia }\end{array}$ \\
\hline 31. & Bouldery & 4010 & NW & 35 & 50 & $\begin{array}{l}\text { Carex stracheyi, Poa alpina, Polygonum } \\
\text { affine, Carex obscura }\end{array}$ \\
\hline 32. & Forest edge & 3680 & $\mathrm{~N}$ & 15 & 5 & $\begin{array}{l}\text { Fragaria nubicola, Galium acutum, Viola } \\
\text { biflora, Cardamine impatiens }\end{array}$ \\
\hline
\end{tabular}

Table 2:-Community types, their distribution and major associates

\begin{tabular}{|c|c|c|c|c|}
\hline Community type & SR & $\begin{array}{l}\text { Habitat } \\
\text { type/s }\end{array}$ & $\begin{array}{l}\text { Altitudinal } \\
\text { range }(\mathbf{m})\end{array}$ & Major associates \\
\hline Carex obscura & 1 & 6 & 3875 & $\begin{array}{l}\text { Dactylis glomerata, Poa pratensis, Circaea } \\
\text { alpina, Galium acutum }\end{array}$ \\
\hline Carex stracheyi & 16 & $\begin{array}{l}1,3,6 \\
7,8,9\end{array}$ & $3740-4075$ & $\begin{array}{l}\text { Danthonia cachemyriana, Polygonum affine, } \\
\text { Carex nubigena, Viola biflora }\end{array}$ \\
\hline $\begin{array}{l}\text { Carex } \\
\text { cachemyriana } \text { mixed }\end{array}$ & 1 & 3 & 3685 & $\begin{array}{l}\text { Cortia depressa, Carex obscura, Trachydium } \\
\text { roylei, Cyananthus lobatus }\end{array}$ \\
\hline $\begin{array}{l}\text { Danthonia cachemyriana-Cortia } \\
\text { depressa-Carex stracheyi mixed }\end{array}$ & 1 & 8 & 3680 & $\begin{array}{l}\text { Dactylis glomerata, Cyananthus lobatus, } \\
\text { Trachydium roylei, Bupleurum lanceolatum }\end{array}$ \\
\hline Danthonia cachemyriana & 3 & $3,4,8$ & $3685-3850$ & $\begin{array}{l}\text { Carex stracheyi, Carex nubigena, } \\
\text { Trachydium roylei, Cyananthus lobatus }\end{array}$ \\
\hline $\begin{array}{l}\text { Danthonia cachemyriana-Carex } \\
\text { stracheyi mixed }\end{array}$ & 6 & 1,3 & $3675-3850$ & $\begin{array}{l}\text { Carex obscura, Trachydium roylei, Cortia } \\
\text { depressa, Dactylis glomerata }\end{array}$ \\
\hline Danthonia $\quad$ cachemyriana- & 1 & 9 & 3925 & Polygonum affine, Gentiana kurroo, \\
\hline
\end{tabular}




\begin{tabular}{|l|l|l|l|l|}
\hline Picrorhiza kurrooa mixed & & & & Fragaria nubicola, Saxifraga parnassifolia \\
\hline $\begin{array}{l}\text { Danthonia cachemyriana- } \\
\text { Polygonum affine mixed }\end{array}$ & 1 & 9 & 4050 & $\begin{array}{l}\text { Anaphalis contorta, Cerastium cerastioides, } \\
\text { Thalictrum chelidonii, Bergenia stracheyi }\end{array}$ \\
\hline $\begin{array}{l}\text { Fragaria nubicola-Galium acutum- } \\
\text { Viola biflora-Cardamine impatiens } \\
\text { mixed }\end{array}$ & 1 & 4 & 3680 & $\begin{array}{l}\text { Impatiens racemosa, Carex obscura, Lactuca } \\
\text { dissecta, Polygonum polystachyum }\end{array}$ \\
\hline Polygonum polystachyum & 1 & 2 & 3715 & $\begin{array}{l}\text { Rumex nepalensis, Fragaria nubicola, } \\
\text { Hackelia uncinata, Elsholtzia eriostachys }\end{array}$ \\
\hline
\end{tabular}

Abbreviations used: SR=Site representation; $1=$ Bouldery; $2=$ Camping site; $3=$ Dry; $4=$ Forest edge; $6=$ Riverine;

$7=$ Rocky; $8=$ Shady moist $9=$ Shrubberries

Table 3:-Community wise distribution of species richness, total density and species diversity

\begin{tabular}{|l|l|l|l|l|}
\hline S.N. & Community type & $\begin{array}{l}\text { Species } \\
\text { richness }\end{array}$ & $\begin{array}{l}\text { Total density } \\
\left(\mathbf{I n d m}^{-2}\right)\end{array}$ & $\begin{array}{l}\text { Diversity } \\
\left(\mathbf{H}^{\prime}\right)\end{array}$ \\
\hline 1. & Carex obscura & 42 & 403.95 & 1.84 \\
\hline 2. & Carex stracheyi & 141 & 660.24 & 1.39 \\
\hline 3. & Carex stracheyi-Danthonia cachemyriana mixed & 40 & 1767.20 & 1.65 \\
\hline 4. & $\begin{array}{l}\text { Danthonia cachemyriana-Cortia depressa-Carex } \\
\text { stracheyi mixed }\end{array}$ & 40 & 1562.10 & 1.68 \\
\hline 5. & Danthonia cachemyriana & 87 & 1004.60 & 1.59 \\
\hline 6. & Danthonia cachemyriana-Carex stracheyi mixed & 124 & 1687.38 & 1.54 \\
\hline 7. & Danthonia cachemyriana-Picrorhiza kurrooa mixed & 21 & 78.65 & 2.25 \\
\hline 8. & Danthonia cachemyriana-Polygonum affine mixed & 14 & 67.80 & 2.11 \\
\hline 9. & $\begin{array}{l}\text { Fragaria nubicola-Galium acutum-Viola biflora- } \\
\text { Cardamine impatiens mixed }\end{array}$ & 87.70 & 2.62 \\
\hline 10. & Polygonum polystachyum & 6 & 26.95 & 1.40 \\
\hline
\end{tabular}

Appendix I:-Diversity, nativity and endemism of vascular plants in Latakharak alpine meadows

\begin{tabular}{|c|c|c|c|}
\hline Family/taxa & $\mathbf{L F}$ & Nativity & Endemism \\
\hline \multicolumn{4}{|l|}{ Angiosperms } \\
\hline \multicolumn{4}{|l|}{ Alliaceae } \\
\hline Allium humile Kunth. & $\mathrm{H}$ & Ind Or & - \\
\hline A. stracheyi Baker & $\mathrm{H}$ & Reg Himal & $\mathrm{E}$ \\
\hline A. wallichii Kunth. & $\mathrm{H}$ & Europe Oriens & - \\
\hline \multicolumn{4}{|l|}{ Apiaceae } \\
\hline Angelica glauca Edgew. & $\mathrm{H}$ & Reg Himal & $\mathrm{E}$ \\
\hline Bupleurum candollei Wall. ex DC. & $\mathrm{H}$ & Reg Himal & - \\
\hline B. falcatum $\mathrm{L}$ & $\mathrm{H}$ & $\begin{array}{l}\text { Europe Oriens As Bor Reg } \\
\text { Himal }\end{array}$ & - \\
\hline B. lanceolatum Wall. ex DC. & $\mathrm{H}$ & Reg Himal & NE \\
\hline Carum carvi $L$. & $\mathrm{H}$ & Europe Oriens As Bor & - \\
\hline Cortia depressa (Don) Norman & $\mathrm{H}$ & Reg Himal & E \\
\hline Heracleum brunonis Benth. & $\mathrm{H}$ & Reg Himal & NE \\
\hline Pleurospermum angelicoides (DC.) Cl. & $\mathrm{H}$ & Reg Himal & - \\
\hline P. densiflorum (Lindl.) Cl. & $\mathrm{H}$ & Reg Himal & $\mathrm{E}$ \\
\hline Selinum elatum (Edgew.) Hiroe & $\mathrm{H}$ & Reg Himal & NE \\
\hline S. vaginatum $\mathrm{Cl}$. & $\mathrm{H}$ & Reg Himal & - \\
\hline Seseli sibiricum (L.) Boiss. & $\mathrm{H}$ & Europe Orient & - \\
\hline Trachydium roylei Lindl. & $\mathrm{H}$ & Reg Himal & - \\
\hline \multicolumn{4}{|l|}{ Asteraceae } \\
\hline $\begin{array}{l}\text { Anaphalis busua (Buch.-Ham. ex D. Don) } \\
\text { DC. }\end{array}$ & $\mathrm{H}$ & Reg Himal & - \\
\hline A. contorta (D. Don) Hk.f. & $\mathrm{H}$ & Reg Himal & - \\
\hline A. nepalensis (Spreng.) Hand.-Mazz. & $\mathrm{H}$ & Reg Himal & - \\
\hline
\end{tabular}




\begin{tabular}{|c|c|c|c|}
\hline A. royleana DC. & $\mathrm{H}$ & Reg Himal & - \\
\hline A. triplinervis (Sims.) Cl. & $\mathrm{H}$ & Reg Himal & - \\
\hline Aster diplostephioides (DC.) Cl. & $\mathrm{H}$ & Reg Himal & - \\
\hline Cirsium wallichii DC. & $\mathrm{H}$ & Reg Himal & $\mathrm{NE}$ \\
\hline $\begin{array}{l}\text { Cremanthodium arnecoides (Wall. ex DC.) } \\
\text { Good }\end{array}$ & $\mathrm{H}$ & Reg Himal & - \\
\hline Dubyaea hispida (D. Don) DC. & $\mathrm{H}$ & Reg Himal & - \\
\hline Gerbera gossypina (Royle) Beauv. & $\mathrm{H}$ & Reg Himal & $\mathrm{NE}$ \\
\hline Inula grandiflora Willd. & $\mathrm{H}$ & Reg Himal et Caucas & - \\
\hline $\begin{array}{l}\text { Jurinella macrocephala (Royle) Aswal et } \\
\text { Goel }\end{array}$ & $\mathrm{H}$ & Reg Himal & $\mathrm{NE}$ \\
\hline Lactuca dissecta D. Don & $\mathrm{H}$ & Reg Himal & - \\
\hline L. macrorhiza (Royle) Hk. f. & $\mathrm{H}$ & Reg Himal & - \\
\hline L. violaefolia (Decne) Cl. & $\mathrm{H}$ & Reg Himal & - \\
\hline L. himalayanum DC. & $\mathrm{H}$ & Europe Reg Himal & - \\
\hline Saussurea costus (Falc.) Lipsch. & $\mathrm{H}$ & Reg Himal & - \\
\hline S. deltoidea $\mathrm{Cl}$ & $\mathrm{H}$ & Reg Himal & - \\
\hline S. obvallata Wall. & $\mathrm{H}$ & Reg Himal & - \\
\hline Senecio alatus Wall. ex DC. & $\mathrm{H}$ & Reg Himal & $\mathrm{NE}$ \\
\hline S. chrysanthemoides DC. & $\mathrm{H}$ & Reg Himal & - \\
\hline S. graciliflorus DC. & $\mathrm{H}$ & Reg Himal & $\mathrm{NE}$ \\
\hline S. kunthianus Wall. ex DC. & $\mathrm{H}$ & Reg Himal & $\mathrm{NE}$ \\
\hline S. quinquelobus Hk.f. \& Th. & $\mathrm{H}$ & Reg Himal & - \\
\hline Solidago virga-aurea $\mathrm{L}$. & $\mathrm{H}$ & Reg Bor Temp & - \\
\hline Tanacetum tomentosum DC. & $\mathrm{H}$ & Reg Himal & $\mathrm{NE}$ \\
\hline Taraxacum officinalis Webber & $\mathrm{H}$ & Reg Temp Bor et Austr & - \\
\hline \multicolumn{4}{|l|}{ Balsaminaceae } \\
\hline Impatiens racemosa DC. & $\mathrm{H}$ & Reg Himal & $\mathrm{NE}$ \\
\hline I. thomsonii Hk. f. & $\mathrm{H}$ & Reg Himal & $\mathrm{NE}$ \\
\hline \multicolumn{4}{|l|}{ Boraginaceae } \\
\hline Arnebia benthamii (Wall. ex G. Don) John. & $\mathrm{H}$ & Reg Himal & $\mathrm{NE}$ \\
\hline Eritrichum canum (Benth.) Kitamura & $\mathrm{H}$ & Ind Or (Reg Himal) & $\mathrm{NE}$ \\
\hline Hackelia uncinata (Royle ex Benth.) Fisch. & $\mathrm{H}$ & Reg Himal & - \\
\hline Maharanga emodi (Wall.) DC. & $\mathrm{H}$ & Reg Himal & $\mathrm{NE}$ \\
\hline \multicolumn{4}{|l|}{ Brassicaceae } \\
\hline Arabidopsis himalaica (Edgew.) Schultz. & $\mathrm{H}$ & Reg Himal & $\mathrm{NE}$ \\
\hline A. thaliana (L.) Heynh. & $\mathrm{H}$ & Reg Temp & - \\
\hline Cardamine impatiens L. & $\mathrm{H}$ & Reg Himal & - \\
\hline Megacarpaea polyandra Benth. & $\mathrm{H}$ & Reg Himal & $\mathrm{NE}$ \\
\hline Thlapsi andersonii (Hk.f. \& Th.) Schulz. & $\mathrm{H}$ & Europe As Bor & - \\
\hline T. arvense $L$. & $\mathrm{H}$ & Europe As Bor & - \\
\hline \multicolumn{4}{|l|}{ Campanulaceae } \\
\hline Campanula colorata Wall. & $\mathrm{H}$ & Ind Or Afghan & - \\
\hline Cyananthus lobatus Wall. ex Benth. & $\mathrm{H}$ & Reg Himal & - \\
\hline \multicolumn{4}{|l|}{ Caprifoliaceae } \\
\hline Lonicera obovata Royle ex Hk. f. \& Th. & $\mathrm{Sh}$ & Reg Himal & - \\
\hline \multicolumn{4}{|l|}{ Caryophyllaceae } \\
\hline Arenaria festucoides Benth. & $\mathrm{H}$ & Reg Himal & $\mathrm{NE}$ \\
\hline Cerastium cerastioides (L.) Britton & $\mathrm{H}$ & Reg Himal & NE \\
\hline Silene gonosperma (Rupt.) Bocqet. & $\mathrm{H}$ & Reg Himal & - \\
\hline S. setisperma Majumdar & $\mathrm{H}$ & Reg Himal & $\mathrm{NE}$ \\
\hline Stellaria decumbens Edgew. & $\mathrm{H}$ & Reg Himal & - \\
\hline \multicolumn{4}{|l|}{ Crassulaceae } \\
\hline Rhodiola bupleuroides Wall. ex Hk. f. \& Th. & $\mathrm{H}$ & Reg Himal & - \\
\hline
\end{tabular}




\begin{tabular}{|c|c|c|c|}
\hline R. heterodonta (Hk.f. \& Th.) Boiss. & $\mathrm{H}$ & Reg Himal & $\mathrm{NE}$ \\
\hline Sedum ewersii Ledeb & $\mathrm{H}$ & Reg Himal & - \\
\hline S. multicaule Wall. ex Lindl. & $\mathrm{H}$ & Reg Himal China & - \\
\hline \multicolumn{4}{|l|}{ Cyperaceae } \\
\hline Carex nivalis Boott & $\mathrm{H}$ & Reg Himal & $\mathrm{NE}$ \\
\hline C. nubigena D. Don & $\mathrm{H}$ & Ind Or & - \\
\hline C. obscura Nees & $\mathrm{H}$ & Reg Himal & NE \\
\hline C. setosa Boott & $\mathrm{H}$ & Reg Himal & - \\
\hline C. stracheyi Boott ex Cl. & $\mathrm{H}$ & Reg Himal & $\mathrm{NE}$ \\
\hline Kobresia duthiei Cl. ex Hk. f. & $\mathrm{H}$ & Reg Himal & E \\
\hline K. laxa Nees & $\mathrm{H}$ & Reg Himal & - \\
\hline \multicolumn{4}{|l|}{ Dipsacaceae } \\
\hline Morina longifolia Wall. ex DC. & $\mathrm{H}$ & Reg Himal & $\mathrm{NE}$ \\
\hline \multicolumn{4}{|l|}{ Ericaceae } \\
\hline Cassiope fastigiata (Wall.) D. Don & Sh & Reg Himal & $\mathrm{NE}$ \\
\hline Rhododendron anthopogon D. Don & $\mathrm{Sh}$ & As Bor Reg Himal & $\mathrm{NE}$ \\
\hline R. campanulatum D. Don & Sh & Reg Himal & NE \\
\hline \multicolumn{4}{|l|}{ Euphorbiaceae } \\
\hline Euphorbia pilosa L. & $\mathrm{H}$ & Europe As Bor & - \\
\hline E. stracheyi Boiss. & $\mathrm{H}$ & Reg Himal & - \\
\hline \multicolumn{4}{|l|}{ Fabaceae } \\
\hline Hedysarum kumaonense Benth. ex Baker & $\mathrm{H}$ & Ind Or (Reg Himal) & $\mathrm{NE}$ \\
\hline Thermopsis barbata Royle & $\mathrm{H}$ & Reg Himal & - \\
\hline Trigonella emodi Benth. & $\mathrm{H}$ & Reg Himal & - \\
\hline \multicolumn{4}{|l|}{ Fumariaceae } \\
\hline Corydalis cashmiriana Duthie \& Prain & $\mathrm{H}$ & Reg Himal & NE \\
\hline C. govaniana Wall. & $\mathrm{H}$ & Reg Himal & $\mathrm{NE}$ \\
\hline C. thyrsiflora Prain & $\mathrm{H}$ & Reg Himal & $\mathrm{NE}$ \\
\hline \multicolumn{4}{|l|}{ Gentianaceae } \\
\hline Gentiana argentea (D. Don) Cl. & $\mathrm{H}$ & Reg Himal China & - \\
\hline G. carinata Griseb. & $\mathrm{H}$ & Reg Himal & $\mathrm{NE}$ \\
\hline G. kurroo Royle & $\mathrm{H}$ & Reg Himal & $\mathrm{NE}$ \\
\hline $\begin{array}{l}\text { Gentianella moorcroftiana (Wall. ex Griseb.) } \\
\text { Shaw ex Rau }\end{array}$ & $\mathrm{H}$ & Reg Himal & $\mathrm{NE}$ \\
\hline G. pedunculata (D. Don) Sm. & $\mathrm{H}$ & Reg Bor Arct & - \\
\hline Halenia elliptica D. Don & $\mathrm{H}$ & Reg Himal & - \\
\hline Lomatogonium carinthiacum (Wulf.) $\mathrm{Br}$. & $\mathrm{H}$ & Europe As Bor & - \\
\hline Swertia angustifolia Buch.-Ham. & $\mathrm{H}$ & Reg Himal & - \\
\hline S. ciliata (D. Don) Burtt. & $\mathrm{H}$ & Reg Himal & $\mathrm{NE}$ \\
\hline S. cuneata Wall. ex D. Don & $\mathrm{H}$ & Reg Himal & $\mathrm{NE}$ \\
\hline S. paniculata Wall. & $\mathrm{H}$ & Reg Himal & $\mathrm{NE}$ \\
\hline \multicolumn{4}{|l|}{ Geraniaceae } \\
\hline Geranium wallichianum D. Don ex Sw. & $\mathrm{H}$ & Reg Himal & $\mathrm{NE}$ \\
\hline G. rotundifolium $\mathrm{L}$. & $\mathrm{H}$ & Reg Bor Temp & - \\
\hline \multicolumn{4}{|l|}{ Haemodoraceae } \\
\hline Aletris pauciflora (Klotz.) Hand.-Mazz. & $\mathrm{H}$ & Reg Himal & - \\
\hline \multicolumn{4}{|l|}{ Iridaceae } \\
\hline Iris kumaonensis D. Don ex Royle & $\mathrm{H}$ & Reg Himal & - \\
\hline \multicolumn{4}{|l|}{ Juncaceae } \\
\hline Juncus concinnus D. Don & $\mathrm{H}$ & Reg Himal & - \\
\hline \multicolumn{4}{|l|}{ Lamiaceae } \\
\hline $\begin{array}{l}\text { Clinopodium umbrosum (Fisch. et May.) } \\
\text { Ktze. }\end{array}$ & $\mathrm{H}$ & Reg BorTemp & - \\
\hline Elsholtzia eriostachys Benth. & $\mathrm{H}$ & Reg Himal & - \\
\hline
\end{tabular}




\begin{tabular}{|c|c|c|c|}
\hline E. fruticosa (D. Don) Rehder & $\mathrm{H}$ & Reg Himal & - \\
\hline Origanum vulgare L. & $\mathrm{H}$ & Europe As et Afr & - \\
\hline Phlomis bracteosa Royle ex Benth. & $\mathrm{H}$ & Reg Himal & - \\
\hline Teucrium royleanum Wall. ex Benth. & $\mathrm{H}$ & Reg Himal & - \\
\hline Thymus linearis Benth. & $\mathrm{H}$ & Europe As et Afr Bor & - \\
\hline \multicolumn{4}{|l|}{ Liliaceae } \\
\hline Fritillaria roylei D. Don ex Hk. & $\mathrm{H}$ & Reg Himal & NE \\
\hline Lloydia serotina (L.) Reichb. & $\mathrm{H}$ & Europe & - \\
\hline Nomocharis nana (Klotzsch) E. H. Wilson & $\mathrm{H}$ & Reg Himal & - \\
\hline N. oxypetala (Royle) Wilson & $\mathrm{H}$ & Reg Himal & $\mathrm{NE}$ \\
\hline Polygonatum cirrhifolium (Wall.) Royle & $\mathrm{H}$ & Reg Himal As Bor & - \\
\hline P. verticillatum (L.) All. & $\mathrm{H}$ & Europe As Bor & - \\
\hline Smilacina purpurea Wall. & $\mathrm{H}$ & Reg Himal & - \\
\hline \multicolumn{4}{|l|}{ Onagraceae } \\
\hline Circaea alpina $\mathrm{L}$. & $\mathrm{H}$ & Europe As Bor Am Bor & - \\
\hline Epilobium latifolium $\mathrm{L}$. & $\mathrm{H}$ & Reg Bor et Arct & - \\
\hline E. roseum (Schreb.) Pers. & $\mathrm{H}$ & Europe As Occ & - \\
\hline \multicolumn{4}{|l|}{ Orchidaceae } \\
\hline Dactylorhiza hatagirea (D. Don) Soo & $\mathrm{H}$ & $\begin{array}{l}\text { Reg Himal Europe Afr Bor } \\
\text { Oriens }\end{array}$ & - \\
\hline Goodyera fusca (Lindl.) Hk.f. & $\mathrm{H}$ & Reg Himal & - \\
\hline Gymnadenia orchidis L. & $\mathrm{H}$ & Reg Himal & $\mathrm{NE}$ \\
\hline Herminium monorchis (L.) R.Br. & $\mathrm{H}$ & Europe As Bor & - \\
\hline Malaxis muscifera (Lindl.) Ktz. & $\mathrm{H}$ & Europe & - \\
\hline Ponerorchis chusua D. Don & $\mathrm{H}$ & Reg Himal & - \\
\hline \multicolumn{4}{|l|}{ Papaveraceae } \\
\hline Meconopsis aculeata Royle & $\mathrm{H}$ & Reg Himal & $\mathrm{NE}$ \\
\hline \multicolumn{4}{|l|}{ Parnassiaceae } \\
\hline Parnassia nubicola Wall. & $\mathrm{H}$ & Reg Himal & $\mathrm{NE}$ \\
\hline P. pussila Wall. & $\mathrm{H}$ & Reg Himal & $\mathrm{NE}$ \\
\hline \multicolumn{4}{|l|}{ Poaceae } \\
\hline Agrostis pilosula Trin. & $\mathrm{H}$ & Ind Or & - \\
\hline Bromus himalaicus Stapf. & $\mathrm{H}$ & Ind Or & - \\
\hline B. japonicus Thunb. & $\mathrm{H}$ & Japan & - \\
\hline Dactylis glomerata L. & $\mathrm{H}$ & Europe As Bor & - \\
\hline Danthonia cachemyriana Faub. \& Spach. & $\mathrm{H}$ & Ind Or (Reg Himal) & $\mathrm{NE}$ \\
\hline Eragrostis nigra Nees ex Steud. & $\mathrm{H}$ & Afr Trop Ind Or Austr & - \\
\hline Poa alpina L. & $\mathrm{H}$ & Reg Bor et Arct Tauria & - \\
\hline P. pratensis L. & $\mathrm{H}$ & Reg Bor Temp & - \\
\hline \multicolumn{4}{|l|}{ Podophyllaceae } \\
\hline Podophyllum hexandrum Royle & $\mathrm{H}$ & Reg Himal & - \\
\hline \multicolumn{4}{|l|}{ Polygonaceae } \\
\hline Oxyria digyna (L.) Hill. & $\mathrm{H}$ & Reg Bor Alp et Arct & - \\
\hline Polygonum affine D. Don & $\mathrm{H}$ & Reg Himal & $\mathrm{NE}$ \\
\hline P. amplexicaule D. Don & $\mathrm{H}$ & Reg Himal & - \\
\hline P. polystachyum Wall. & $\mathrm{H}$ & Reg Himal & - \\
\hline P. rumicifolium Royle ex Bab. & $\mathrm{H}$ & Reg Himal & $\mathrm{NE}$ \\
\hline P. vaccinifolium Wall. ex Meissn. & $\mathrm{Sh}$ & Reg Himal & - \\
\hline Rheum australe D. Don & $\mathrm{H}$ & Reg Himal & $\mathrm{NE}$ \\
\hline R. webbianum Royle & $\mathrm{H}$ & Reg Himal & $\mathrm{NE}$ \\
\hline Rumex nepalensis Spreng. & $\mathrm{H}$ & $\begin{array}{l}\text { As Occ Ind Or Malaya Afr } \\
\text { Austr }\end{array}$ & - \\
\hline R. acetosa $\mathrm{L}$. & $\mathrm{H}$ & Europe As Bor & - \\
\hline Primulaceae & & & \\
\hline
\end{tabular}




\begin{tabular}{|c|c|c|c|}
\hline Androsace sarmentosa Wall. & $\mathrm{H}$ & Reg Himal & $\mathrm{NE}$ \\
\hline Primula atrodentata $\mathrm{Sm}$. & $\mathrm{H}$ & Reg Himal & $\mathrm{NE}$ \\
\hline P. denticulata $\mathrm{Sm}$. & $\mathrm{H}$ & Reg Himal & - \\
\hline P. elliptica Royle & $\mathrm{H}$ & Reg Himal & $\mathrm{NE}$ \\
\hline P. glomerata Pax. & $\mathrm{H}$ & Reg Himal & $\mathrm{NE}$ \\
\hline P. macrophylla D. Don & $\mathrm{H}$ & As et Am Bor & - \\
\hline P. reptans Hk.f. ex Wall. & $\mathrm{H}$ & Reg Himal & $\mathrm{NE}$ \\
\hline \multicolumn{4}{|l|}{ Ranunculaceae } \\
\hline Aconitum voilaceum Jacq. & $\mathrm{H}$ & Reg Himal & $\mathrm{NE}$ \\
\hline A. balfourii Stapf. & $\mathrm{H}$ & Reg Himal & $\mathrm{NE}$ \\
\hline A. heterophyllum Wall. ex Royle & $\mathrm{H}$ & Reg Himal & $\mathrm{NE}$ \\
\hline Anemone obtusiloba D. Don & $\mathrm{H}$ & Reg Himal & - \\
\hline A. polyanthes D. Don & $\mathrm{H}$ & Reg Himal & $\mathrm{NE}$ \\
\hline A. rupicola Camb. & $\mathrm{H}$ & Reg Himal & - \\
\hline Aquilegia pubiflora Wall. ex Royle & $\mathrm{H}$ & Ind Or & - \\
\hline Caltha palustris $\mathrm{L}$. & $\mathrm{H}$ & Reg Bor Temp et Arct & - \\
\hline Clematis acuminata DC. & Sh & Reg Himal & $\mathrm{NE}$ \\
\hline C. barbellata Edgew. & Sh & Reg Himal & $\mathrm{NE}$ \\
\hline Oxygraphis polypetala (Royle) Hk.f. \& Th. & $\mathrm{H}$ & Reg Himal & $\mathrm{NE}$ \\
\hline $\begin{array}{l}\text { Paraquilegia microphylla (Royle) Drum. \& } \\
\text { Hutch. }\end{array}$ & $\mathrm{H}$ & Reg Himal Sibir Altaic & - \\
\hline Ranunculus hirtellus Royle & $\mathrm{H}$ & Reg Himal & - \\
\hline Thalictrum alpinum $\mathrm{L}$. & $\mathrm{H}$ & Reg Bor et Arct & - \\
\hline T. chelidonii DC. & $\mathrm{H}$ & Reg Himal & - \\
\hline T. elegans Wall. ex Royle & $\mathrm{H}$ & Reg Himal & $\mathrm{NE}$ \\
\hline T. pauciflorum Royle & $\mathrm{H}$ & Europe et As Bor & - \\
\hline T. reniforme Wall. & $\mathrm{H}$ & Reg Himal & $\mathrm{NE}$ \\
\hline \multicolumn{4}{|l|}{ Rosaceae } \\
\hline Arunchus dioicus (Walt.) Fernald & $\mathrm{H}$ & Reg Bor Temp & - \\
\hline Cotoneaster microphyllus Wall. ex Lindl. & Sh & Reg Himal & - \\
\hline Fragaria nubicola Lindl. ex Lacaita & $\mathrm{H}$ & Am Bor & - \\
\hline Geum elatum Wall. & $\mathrm{H}$ & Reg Himal & $\mathrm{NE}$ \\
\hline Potentilla argyrophylla Wall. ex Lehm. & $\mathrm{H}$ & Reg Himal & $\mathrm{NE}$ \\
\hline P. atrosanguinea Lodd. & $\mathrm{H}$ & Reg Himal & $\mathrm{NE}$ \\
\hline P. microphylla D. Don & $\mathrm{H}$ & Reg Himal & $\mathrm{NE}$ \\
\hline P. peduncularis D. Don & $\mathrm{H}$ & Reg Himal & - \\
\hline Rosa macrophylla Lindl. & Sh & Reg Himal China & - \\
\hline Sibaldia cuneata Hornem. ex Kunge & $\mathrm{H}$ & Reg Bor et Austr & - \\
\hline S. purpurea Royle & $\mathrm{H}$ & Reg Himal & $\mathrm{NE}$ \\
\hline \multicolumn{4}{|l|}{ Rubiaceae } \\
\hline Galium acutum Edgew. & $\mathrm{H}$ & Reg Himal & $\mathrm{NE}$ \\
\hline G. elegans Wall. ex Roxb. & $\mathrm{H}$ & Europe As Temp & - \\
\hline \multicolumn{4}{|l|}{ Salicaceae } \\
\hline Salix lidleyana Wall. ex Ander. & Sh & Reg Himal & - \\
\hline \multicolumn{4}{|l|}{ Saxifragaceae } \\
\hline Bergenia stracheyi (Hk.f. \& Th.) Engl. & $\mathrm{H}$ & Reg Himal & $\mathrm{NE}$ \\
\hline Chrysosplenium carnosum Hk.f. \& Th. & $\mathrm{H}$ & Reg Himal & $\mathrm{NE}$ \\
\hline $\begin{array}{l}\text { Saxifraga brachypoda } \\
\text { Frimbriata (DC.) Engl. }\end{array}$ & $\mathrm{H}$ & Reg Himal & - \\
\hline S. parnassifolia D. Don & $\mathrm{H}$ & Reg Himal & $\mathrm{NE}$ \\
\hline S. pulvinaria $\mathrm{Sm}$. & $\mathrm{H}$ & Reg Himal & $\mathrm{NE}$ \\
\hline S. sibirica L. & $\mathrm{H}$ & As Bor et Arct & - \\
\hline \multicolumn{4}{|l|}{ Scrophulariaceae } \\
\hline Euphrasia himalayica Wettst. & $\mathrm{H}$ & Reg Himal & $\mathrm{NE}$ \\
\hline
\end{tabular}




\begin{tabular}{|c|c|c|c|}
\hline Pedicularis bicornuta Klotz. & $\mathrm{H}$ & Reg Himal & $\mathrm{NE}$ \\
\hline P. hoffmeisteri Klotz. \& Garcke & $\mathrm{H}$ & Reg Himal & $\mathrm{NE}$ \\
\hline P. hookeriana Wall. ex Kunth. & $\mathrm{H}$ & Reg Himal Persea & - \\
\hline P. pectinata Wall. & $\mathrm{H}$ & Reg Himal & $\mathrm{NE}$ \\
\hline Picrorhiza kurrooa Royle ex Benth. & $\mathrm{H}$ & Reg Himal & - \\
\hline Scrophularia himalensis Royle ex Benth. & $\mathrm{H}$ & Reg Himal & - \\
\hline Verbascum thapsus L. & $\mathrm{H}$ & Europe Or Reg Himal & - \\
\hline Veronica capitata Benth. & $\mathrm{H}$ & Reg Himal & - \\
\hline V. lanosa Royle ex Benth. & $\mathrm{H}$ & Reg Himal Afghan & $\mathrm{NE}$ \\
\hline \multicolumn{4}{|l|}{ Valerianaceae } \\
\hline Nardostachys grandiflora DC. & $\mathrm{H}$ & Reg Himal & - \\
\hline Valeriana hardwickii Wall. & $\mathrm{H}$ & Reg Himal Malaya & - \\
\hline$V$. pyrolaefolia Decne & $\mathrm{H}$ & Reg Himal & - \\
\hline \multicolumn{4}{|l|}{ Violaceae } \\
\hline Viola biflora $\mathrm{L}$. & $\mathrm{H}$ & Reg Bor Temp & - \\
\hline \multicolumn{4}{|l|}{ Gymnosperms } \\
\hline \multicolumn{4}{|l|}{ Cupressaceae } \\
\hline Juniperus communis L. & Sh & Reg Bor Temp et Arct & - \\
\hline J. indica Bertol & $\mathrm{Sh}$ & Soongar Reg Himal Reg Bor & - \\
\hline \multicolumn{4}{|l|}{ Pteridophytes } \\
\hline \multicolumn{4}{|l|}{ Athyriaceae } \\
\hline $\begin{array}{llll}\text { Athyrium attenuatum } & \text { (Cl.) } & \text { Tag. forma } \\
\text { Attenuatum } & & & \\
\end{array}$ & $\mathrm{Pt}$ & - & - \\
\hline A. duthiei (Bedd.) Bedd. & Pt & - & - \\
\hline A. wallichianum Ching & $\mathrm{Pt}$ & - & - \\
\hline Cystopteris fragilis (L.) Bernh. & $\mathrm{Pt}$ & - & - \\
\hline C. montana (Lam.) Bernh. ex Desv. & $\mathrm{Pt}$ & - & - \\
\hline \multicolumn{4}{|l|}{ Cryptogrammaceae } \\
\hline Cryptogramma stellarii (Gmel.) Prantl. & $\mathrm{Pt}$ & - & - \\
\hline \multicolumn{4}{|l|}{ Dryopteridaceae } \\
\hline Dryopteris barbigera (Hk.) Ktze. & $\mathrm{Pt}$ & - & - \\
\hline D. panda (Cl.) Christ. & $\mathrm{Pt}$ & - & - \\
\hline D. wallichiana (Spr.) Hyl. & $\mathrm{Pt}$ & - & - \\
\hline Polystichum lachenense (Hk.) Bedd. & $\mathrm{Pt}$ & - & - \\
\hline P. prescottianum (Wall. ex Mett.) Moore & $\mathrm{Pt}$ & - & - \\
\hline P. wilsonii Christ. & $\mathrm{Pt}$ & - & - \\
\hline \multicolumn{4}{|l|}{ Equisetaceae } \\
\hline Equisetum arvense L. & $\mathrm{Pt}$ & - & - \\
\hline \multicolumn{4}{|l|}{ Osmundaceae } \\
\hline Osmunda claytoniana $\mathrm{L}$. & $\mathrm{Pt}$ & - & - \\
\hline \multicolumn{4}{|l|}{ Polypodiaceae } \\
\hline Lepisorus morrisonensis (Hayata) H. Ito & $\mathrm{Pt}$ & - & - \\
\hline L. tenuipes Ching et Khullar & $\mathrm{Pt}$ & Reg Himal & $\mathrm{E}$ \\
\hline $\begin{array}{l}\text { Phymatopteris stracheyi (Ching) Pichi - } \\
\text { Sermoli }\end{array}$ & $\mathrm{Pt}$ & - & - \\
\hline \multicolumn{4}{|l|}{ Thelypteridaceae } \\
\hline Pseudophegopteris levengii (Cl.) Ching & $\mathrm{Pt}$ & - & - \\
\hline \multicolumn{4}{|l|}{ Woodsiaceae } \\
\hline Woodsia lanosa $\mathrm{Hk}$. & $\mathrm{Pt}$ & - & - \\
\hline
\end{tabular}

Abbreviations used: $\mathrm{H}=\mathrm{Herb} ; \mathrm{Sh}=\mathrm{Shrub}$; $\mathrm{P}=$ Pteridophyte; $\mathrm{LF}=\mathrm{Life}$ form; Afghan= Afghanistan; Afr= Africa; Alp= Alpine; Am= America; Amphig= Amphigaea; Arab= Arabia; Arct = Arctic; As= Asia; Austr $=$ Australia; Baluchist $=$ Baluchistan; Bor $=$ Borealis; Caucas $=$ Caucasus; Centr $=$ Central; Cosmop $=$ Cosmopolitan; et $=$ And; Geront $=$ Gerontia Himal= Himalayan; Hisp= Hispan; Ind= Indian; Mediterr= Mediterranean; Mongol= Mongolia; 
N. Zel $=$ New Zeyland; Occ $=$ Occidentalis; Or $=$ Oriental; Orient $=$ Oriental; Reg= Region; Soongar $=$ Soongarica; Subtrop $=$ Subtropical; Temp $=$ Temperate; Trop $=$ Tropical; Turkist $=$ Turkistan; E $=$ Endemic $; \mathrm{NE}=$ Near Endemic

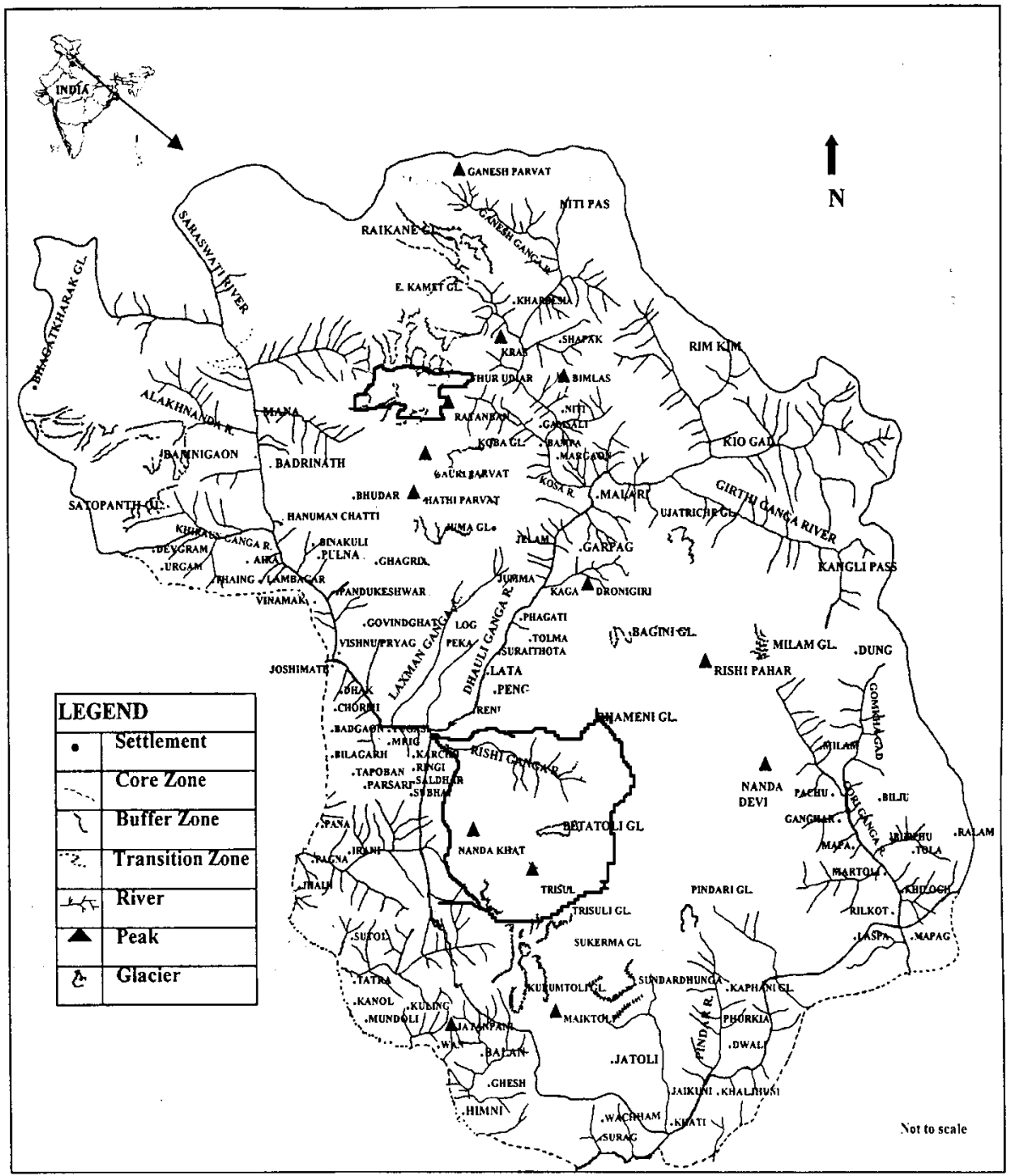

Fig 1:- Location of Nanda Devi Biosphere Reserve (Prepared by Lead Office, GBPIHED, Kosi-Katarmal, Almora) 


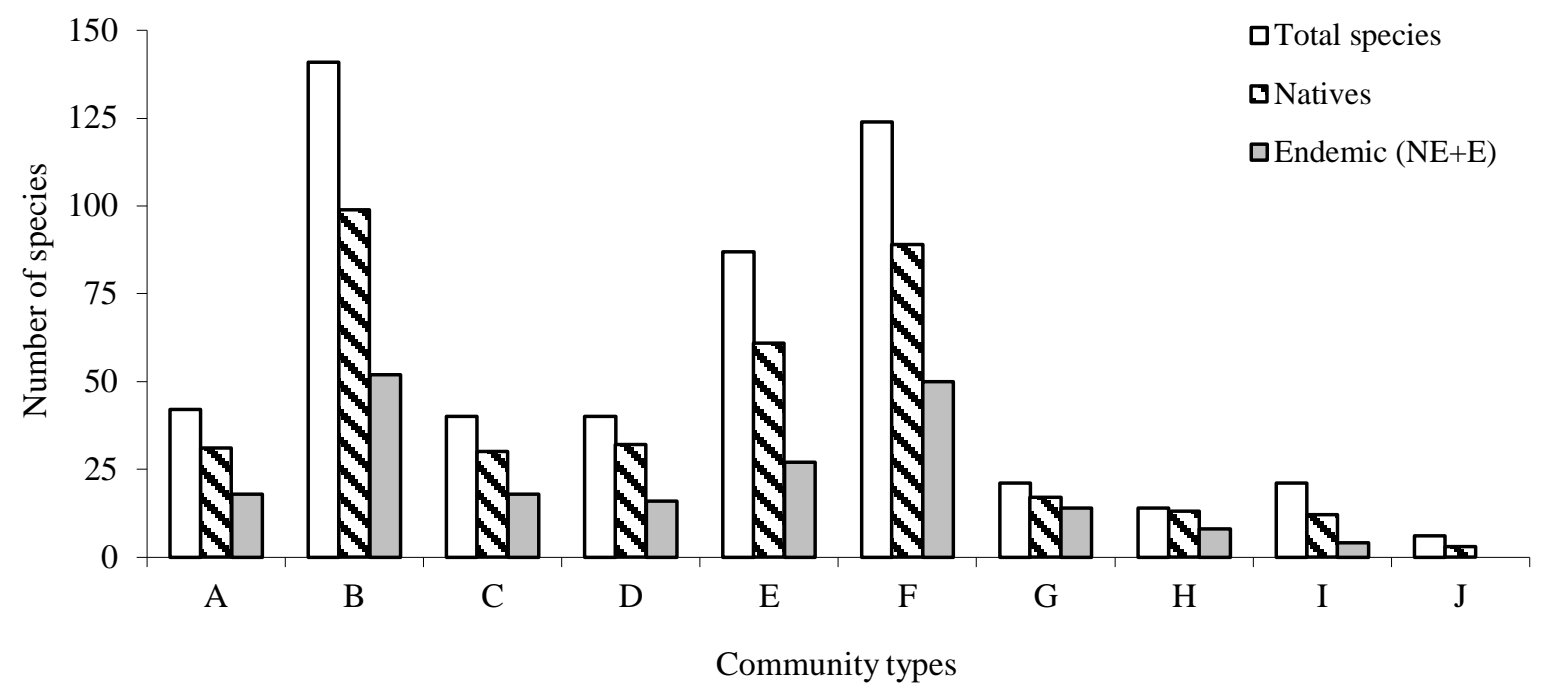

Fig. 2:- Distribution of native and endemic species in alpine communities of Latakharak area

Abbreviations used (Community types A-J):- A=Carex obscura; $\mathrm{B}=$ Carex stracheyi; $\mathrm{C}=$ Carex stracheyiDanthonia cachemyriana mixed; $\mathrm{D}=$ Danthonia cachemyriana-Cortia depressa-Carex stracheyi mixed; $\mathrm{E}=$ Danthonia cachemyriana; $\mathrm{F}=$ Danthonia cachemyriana-Carex stracheyi mixed; $\mathrm{G}=$ Danthonia cachemyrianaPicrorhiza kurrooa mixed; H=Danthonia cachemyriana-Polygonum affine mixed; I=Fragaria nubicola-Galium acutum-Viola biflora-Cardamine impatiens mixed; and $\mathrm{J}=$ Polygonum polystachyum
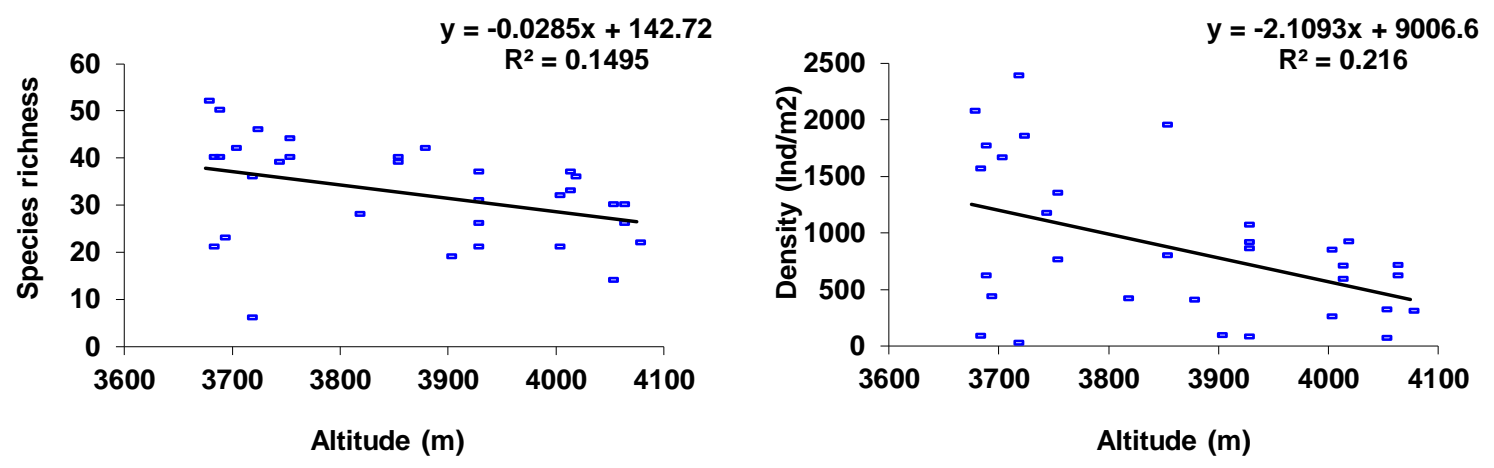

Fig. 3:-Correlations between altitude and species richness; (a) and (b) altitude and density

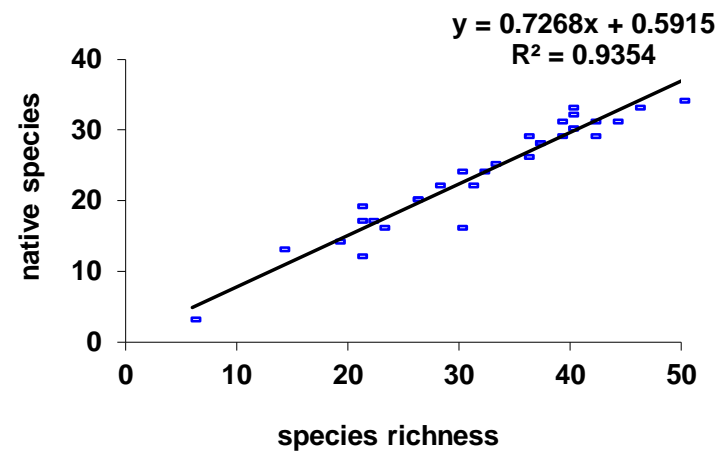

Fig. 4:-Correlations between species richness and native species 


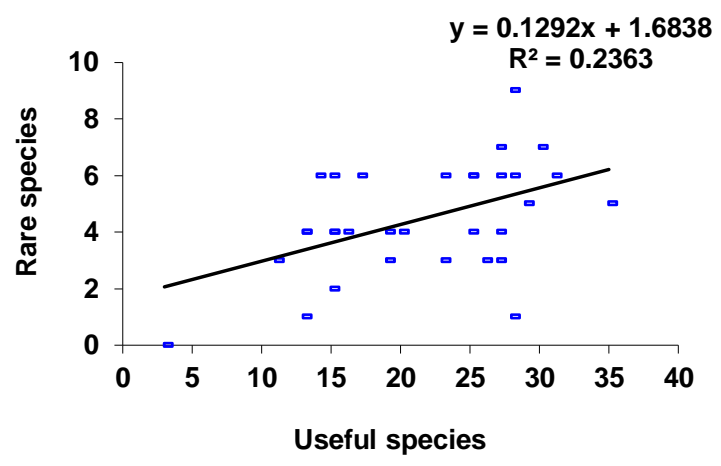

Fig 5:- Correlation between numbers of useful species with number of rare species

\section{Discussion:-}

Total 10 communities and 227 species have been recorded from Latakharak alpine meadows of Nanda Devi Biosphere Reserve. The species and communities of the meadow were analyzed for nativity, endemism, human dependence and rarity. The occurrence of high native, endemic, economically important, and rare-endangered species indicated the high conservation value of the meadows.

Alpine plants are distributed over different microhabitats and altitude with distinct ecological requirements (Rawat \& Uniyal, 1993). In the study area, the distribution of communities and species changes in response to habitats. The tussuck forming grasses and cushion and spreading forbs covers the maximum part of the alpine meadows. Considering all the sites together, most species showed regular distribution pattern both, among sites and communities in all the areas.

The total density range among communities (26.95-1767.20 $\mathrm{Ind}^{-2}$ ) was comparatively higher. Most of these high density were dominated by grasses, sedges, cushion, and spreading forbs indicating the proliferation of these communities in the area. The low density of Polygonum polystachyum, Danthonia cachemyriana-Polygonum affine mixed, Danthonia cachemyriana-Picrorhiza kurrooa mixed, Fragaria nubicola-Galium acutum-Viola bifloraCardamine impatiens mixed communities may be due to high biotic pressures. Some of these communities were found in camping sites and dominated by weeds i.e., Polygonum polystachyum, Rumex nepalensis etc. These are the characteristic species of the camping sites where the organic matter was found accumulated due to the excretory wastes of the animals. Such species do not allow other species to grow. Among the habitats, camping sites, shrubberies and forest edges showed the lowest densities.

The species richness among communities (6-141) was comparatively high when compared to the Panwali Kantha bugyal (5-42) (Raizada et al., 1998). The H' (1.33-3.04) was within the reported range for Valley of Flowers (Kala et al., 1998).

A significant negative correlation had been found between altitude and species richness $(r=0.387 \mathrm{p}<0.01 \mathrm{n}=32)$ and altitude and density $(\mathrm{r}=-0.465, \mathrm{p}<0.01 \mathrm{n}=32)$ in Latakharak alpine meadows indicating that with the increasing altitude the species richness and density decreased. (Fig. 3 a-b).

The occurrence of maximum number of species in Carex stracheyi and Danthonia cachemyriana-Carex stracheyi mixed, community may be attributed due to their wide range of distribution and habitat preference. Similarly, the occurrence of minimum number of species in Polygonum polystachyum and Danthonia cachemyriana-Polygonum affine mixed communities may be due to their narrow range of distribution and restricted habitats.

The Polygonum polystachyum community was represented by camping site, a habitat which have been developed due to the camping by sheep grazers and also by trampling of sheep's and goats (Ram \& Singh, 1994). The low species richness in Polygonum polystachyum and Danthonia cachemyriana-Polygonum affine mixed community was represented by shrubberries habitat. The occurrence of shrubs in the habitat might be the reason of low species diversity. 
The maximum diversity in the communities, such as Fragaria nubicola-Galium acutum-Viola biflora-Cardamine impatiens mixed, Danthonia cachemyriana-Picrorhiza kurrooa mixed, Danthonia cachemyriana-Polygonum affine mixed may be due to heterogeneous composition of the species with almost similar trend of distribution. The minimum diversity in the communities, such as Carex stracheyi, Polygonum polystachyum, Danthonia cachemyriana-Carex stracheyi mixed and Danthonia cachemyriana, may be mainly due to narrow range of distribution, habitat restriction and dominance of some species.

Due to relatively harsh climatic and topographic conditions of the alpine meadows the non-native species could not easily adapted, therefore the existence of no-native species is comparatively low in this region. Further, due to the remoteness, inaccessibility and severe climatic conditions the anthropogenic pressure is low compared to the subtropical and temperate zones of IHR. This has led to the high diversity of native and endemic species.

A positive correlation had been found between species richness and native species among the communities $(0.967$, $\mathrm{p}<0.01 \mathrm{n}=32$ ) (Fig. 4).

Aconitum balfourii, A. heterophyllum, Angelica glauca, Megacarpaea polyandra, Bergenia stracheyi, Dactylorhiza hatagirea, Polygonatum verticillatum, Arnebia benthamii, Picrorhiza kurrooa, Nardostachys grandiflora, Pleurospermum angelicoides, Podophyllum hexandrum, Jurinella macrocephala, Saussurea obvallata, Rheum australe, etc., had caused population depletion to a great extent in the wild. Continuous overexploitation of such species may lead to their early extinction from their natural habitats (Samant et al., 2001). Therefore, timely human interventions for the conservation of these species are urgently required.

A significant positive relationship had been found between the number of useful species and number of rare species Latakharak (0.486, $\mathrm{p}<0.01 \mathrm{n}=32)$, (Fig. 5) indicating that the use of the species was directly proportional to the rarity of the species.

Overall, 10 communities have been identified from the Latakharak alpine meadows of NDBR, which are comparatively higher than the communities identified from Valley of Flowers (Kala et al., 1998), Baideni (Rikhari et al., 1992; Negi et al., 1992) and Panwalikantha, alpine meadows (Raizada et al., 1998). In all the alpine meadows, species richness, diversity, nativity, endemism, rarity, and use values varied from community to community.

Based on these values, in Latakharak alpine meadows, Carex stracheyi (Total species 141; Natives 99; Endemic 52; Rare-endangered 24; Useful species 75); and Danthonia cachemyriana-Carex stracheyi (Total species 124; Natives 89; Endemic 50; Rare-endangered 18; Useful species 71), communities, have been identified as high value communities and merit priority attention for conservation.

The richness of useful species in these communities indicates high anthropogenic pressure. If indiscriminate exploitation of the useful species from these communities continues, there is possibility of extinction of these species from their natural habitats leading to habitat alterations and ecosystem imbalance. Therefore, there is an urgent need to pay adequate attention for the conservation of these communities supporting ecologically and economically important species.

\section{Conclusions:-}

The present study conducted in Latakharak alpine meadows of NDBR provides data base on compositional and structural, distribution pattern of species, communities, native, endemic and rare-endangered species within different communities. The study provides a comprehensive information on human dependence on plant resources, indigenous uses, rare-endangered species, and prioritization of communities for conservation. Occurrence of 10 alpine communities, mostly representative ones and 213 species in the study area suggested its importance from the point view of conservation.

The present study indicated that species richness and density decreased with the increasing altitude. Decreasing trend of the species with the increasing altitude has been also reported in the IHR (Samant \& Dhar, 1997, Samant et al., 1998a, 1998b). Habitats play a significant role in the distribution pattern and growth of the plant species. Therefore, habitat wise assessment of the alpine vegetation has been carried out for the first time in the IHR. The conservation of habitats is most important for the conservation of species. If habitats are conserved, the species present will be conserved automatically. 
The communities with wide range of distribution had high species richness and species diversity compared to the communities with narrow range of distribution. Camping sites were mainly dominated by Rumex nepalensis, Polygonum polystachyum, etc. Proliferation of these species in the area is causing habitat alterations, loss of biodiversity as well as ecosystem imbalance.

Keeping in view the rich biodiversity of alpine meadows it is pertinent to make an appropriate strategy and action plan for the conservation and management of habitats, communities and ecosystems, supporting high value species.

\section{Acknowledgements:-}

The authors are thankful to the Director of the Institute for facilities and encouragement. Help received from Dr. H.C. Joshi during field surveys and preparation of the paper, Dr. K.K. Kothari, Mr. S. Pant and Mr. B.M. Joshi during the preparation of this paper is highly acknowledged. Ministry of Environment \& Forests, New Delhi and Council of Scientific and Industrial Research, New Delhi, are greatly acknowledged for financial assistance.

\section{References:-}

1. Anonymous (1883-1970): Index Kewensis Plantarum Phanerogamarum Vol. 1-2 (1883-1885) and 15 Suppl. (1886-1970). Clarendron Press, Oxford.

2. Anonymous (1989): The State of Forest Report 1989. Forest Survey of India, Government of India, Dehradun.

3. Billings, W.D. (1973): Arctic and alpine vegetation: similarities, differences and susceptibility to disturbance. Biosciences, 23: 697-704.

4. Dhar, U. \& Samant, S.S. (1993): Endemic diversity of Indian Himalaya I. Ranunculaceae and II. Paeoniaceae. Journal of Biogeography, 20: 659-668.

5. Dhar, U., Rawal, R.S. and Samant, S.S. (1997): Structural diversity and representativeness of forest vegetation in a protected area of Kumaun Himalaya, India: implications for conservation. Biodiversity and Conservation, 6: $1045-1062$.

6. Greig-Smith, P. (1957): Quantitative plant ecology. New York: Academic Press.

7. Grime, J.P. (1979): Plant Strategies and Vegetation Processes. New York: John Wiley and Sons.

8. Hajra, P.K. and Balodi, B. (1995): Plant wealth of Nanda Devi Biosphere Reserve. Botanical Survey of India, Calcutta.

9. Hajra, P.K. and Jain, S.K. (1981): A contribution to the Botany of Nanda Devi National Park in Uttar Pradesh, India. Botanical Survey of India, Howrah.

10. Hajra, P.K. (1983): A contribution to the Botany of Nanda Devi National Park. Botanical Survey of India, Howrah.

11. Harper, J.L. (1977): Population Biology of Plants. London: Academic Press.

12. Johnsing, A.J.T., Rawat, G.S., Satyakumar, P.V., and Kuar, J. (1998): Prioritization of area for biodiversity conservation of alpine zone in Trans and Greater Himalaya in India. Biodiversity Conservation Prioritization Project in India. WWF, New Delhi. pp. 212-224.

13. Joshi, H.C., Arya, S.C. and Samant, S.S. (1999): Diversity, distribution and indigenous uses of medicinal and edible plants in a part of Nanda Devi Biosphere Reserve I. Himalayan Biosphere Reserves, 1(1\&2): 49-65.

14. Joshi, H.C., Arya, S.C. and Samant, S.S. (2001): Diversity, distribution and indigenous uses of plant species in Pindari area of Nanda Devi Biosphere Reserve-II. Indian Journal of Forestry, 24 (4): 514-536.

15. Joshi, S.P., Raizada, A. \& Srivastava, M.M. (1988): Net primary productivity of a high altitude grassland in Garhwal Himalaya. Tropical Ecology, 29: 15-20.

16. Joshi, S.P. \& Srivastava, M.M. (1988): Effect of grazing on species composition, diversity and productivity in a high altitude pasture in Garhwal Himalaya. Int. Jour. Enviro. Sci., 14: 221-227.

17. Joshi, S.P. \& Srivastava, M.M. (1991): The status of grazing land of alpine region in Garhwal Himalayas. In: Advances in Himalayan Ecology. (ed.) G.S. Rajwar, Today and Tomorrow Printers and Publishers, New Delhi. pp. 1-12.

18. Joshi, H.C. \& Samant, S.S. (2004): Compositional and structural diversity of woody vegetation in Nanda Devi Biosphere Reserve of west Himalaya I. International Journal of Sustainable Development and World Ecology, 11(3): $326-336$

19. Kala, C.P. and Rawat, G.S. (1999): Effects of livestock grazing on the species diversity and biomass production in the alpine meadows of Garhwal Himalaya, India. Tropical Ecology, 40(1): 69-74.

20. Kala, C.P., Rawat, G.S. and Uniyal, V.K. (1998): Ecology and conservation of the Valley of Flowers National Park, Garhwal Himalaya. Report. Wildlife Institute of India, Dehradun. 
21. Kaul, V. and Sarin, Y.K. (1971): The phytosociology of some alpine meadows in North West Himalaya. Vegetatio, 23: 261-368.

22. Kersaw, K.A. (1973): Quantitative and dynamic plant ecology. Second edition. London: Edward Arnold Limited.

23. Ludwig, J.A. and Reynolds, J.F. (1988): Statistical Ecology: A primer on methods and Computing. USA: John Willey and Sons, $337 \mathrm{pp}$.

24. Mani, M.S. (1978): Ecology and Phytogeography of the High Altitude Plants of the North West Himalaya. New Delhi: Oxford and IBH Publishing Company, India.

25. Misra, R. (1968): Ecological Work Book. Calcutta: Oxford and IBH Publishing Company.

26. Mueller-Dombois, D. and Ellenberge, H. (1974): Aims and methods of vegetation ecology. New York: John Willey and Sons.

27. Naithani, B.D. (1984 and 1985): Flora of Chamoli district. Vol. I and II. Botanical Survey of India, Howrah.

28. Nautiyal, B.P., Pandey, N. and Bhatt, A.D. (1997): Analysis of vegetation pattern in alpine zone in North West Himalaya: A case study of Garhwal Himalaya with special reference to diversity and distributional patterns. International Journal of Ecology and Environmental Science, 23: 49-65.

29. Nautiyal, M.C., Nautiyal, B.P. and Prakash, V. (2001): Phenology and growth form distribution in an alpine pasture at Tungnath, Garhwal, Himalaya. Mountain Research and Development, 21(2): 168-174.

30. Nayar, M.P. and Sastry, A.R.K. (1987,1988, 1990): Red Data Book of Indian Plants. Vol. I-III. Botanical Survey of India, Calcutta.

31. Negi, C.G.S., Rikhari, H.C. and Singh, S.P. (1992): Phenological features in relation to growth forms and biomass accumulation in an alpine meadow of the central Himalaya. Vegetatio, 101: 161-170.

32. Negi, G.C.S, Rikhari, H.C. and Singh, S.P. (1993): Plant re-growth following selective horse and sheep grazing and clipping in an Indian central Himalayan alpine meadow. Arctic and Alpine Research, 25(3): 211-215.

33. Pangtey, Y.P.S., Rawal, R.S., Bankoti, N.S. and Samant, S.S. (1990): Phenology of high altitude plants of Kumaun in central Himalaya, India. International Journal of Biometeorology, 34: 122-127.

34. Pangtey, Y.P.S., Samant, S.S. and Rawal, R.S. (1993): Enumeration of ferns of Pindari, Sarju and east Ramganga valleys of Kumaun (western Himalaya). Higher Altitude of Indian Sub continent. Additional Series of Indian Journal of Forestry, 4: 119-152.

35. Polunin, O. and Stainton, A. (1984): Flowers of the Himalaya. Oxford: Oxford University Press.

36. Raizada, A., Joshi, S.P. and Srivastava, M.M. (1998): Composition and Vegetational diversity in an alpine grassland in the Garhwal Himalayas. Tropical Ecology, 39: 133-141.

37. Ram, J. (1988): Phytosociology and Primary Productivity of an Alpine Grassland of Garhwal Himalaya. Ph.D. Thesis, Kumaun University, Nainital.

38. Ram, J. (1992): Effect of clipping on aboveground plant biomass and total herbage yield in a grassland above treeline in central Himalaya, India. Arctic and Alpine Research, 24: 78-81.

39. Ram, J. \& Singh, S.P. (1994): Ecology and Conservation of alpine meadows in Central Himalaya. India. In Y.P.S. Pangtey \& R. S. Rawal (eds.) High altitudes of the Himalaya: biogeography, ecology and conservation. Gyanodaya Prakashan, Nainital. pp. 33-35.

40. Ram, J., Singh, S.P. and Singh, J.S. (1988): Community level phenology of grassland above treeline in Central Himalaya, India. Arctic and Alpine Research, 20: 325-332.

41. Ram, J., Singh, J.S. \& Singh, S.P. (1989): Plant biomass, species diversity and net primary production in a central Himalayan high altitude grassland. Journal of Ecology, 77: 456-468.

42. Rau, M.A. (1975): High altitude flowering plants of western Himalaya. Botanical Survey of India, Calcutta.

43. Rawat, G.S. and Pangtey, Y.P.S. (1987): A contribution to the ethnobotany of alpine regions of Kumaun. Journal of Economic and Taxonomic Botany, 11(1): 139-148.

44. Rawat, G.S. and Pangtey, Y.P.S. (1987): Floristic structure of snowline vegetation in central Himalaya, India. Arctic and Alpine Research, 19 (2): 195-201.

45. Rawat, G.S. and Rodgers, W.A. (1988): The alpine meadows of Uttar Pradesh. An Ecological review. In Pathak, P.C. (ed.), Rangelands: Resource and Management. Jhansi. 119-137.

46. Rawat, G.S., and Uniyal, V.K. (1993): Pastoralism and plant conservation. The Valley of Flowers dilemma. Environmental Conservation, 20: 164-167.

47. Rikhari, H.C., Negi, G.C.S., Rana, B.S., and Singh, S.P. (1992): Phytomass and primary productivity in several communities of a central Himalayan alpine meadow, India. Arctic and Alpine Research, 24: 344-351.

48. Rodgers, W.A. and Panwar, H.S. (1988): Planning a Wildlife Protected Area Network in India. Vol. I. Report. Wildlife Institute of India, Dehradun. 
49. Sahai, B. and Kimothi, M.M. (1996): Remote sensing for surveying, mapping and monitoring of conservation areas in Himalaya. In Ramakrishnan, P.S., Purohit, A.N., Saxena, K.G., Rao, K.S. and Maikhuri, R.K. (eds.), Conservation and management of biological resources in Himalaya. New Delhi: Oxford and IBH Publication, 233-258 pp.

50. Samant, S.S. (1993): Diversity and status of plants in Nanda Devi Biosphere Reserve. In Report, Army Head Quarters, Scientific and Ecological Expedition to Nanda Devi., New Delhi: 54-85 pp.

51. Samant, S.S. (1999): Diversity, nativity and endemism of vascular plants in a part of Nanda Devi Biosphere Reserve in west Himalaya I. Himalayan Biosphere Reserves, 1(1\&2): 1-28.

52. Samant, S.S. and Dhar, U. (1997): Diversity, endemism and economic potential of wild edible plants of Indian Himalaya. International Journal of Sustainable Development and World Ecology, 4: 179-191.

53. Samant, S.S., Dhar, U. and Rawal, R.S. (1996a): Natural resource use by some natives within Nanda Devi Biosphere Reserve in west Himalaya. Ethnobotany, 8: 40-50.

54. Samant, S.S., Dhar, U. and Rawal, R.S. (1996b): Conservation of rare endangered plants: The context of Nanda Devi Biosphere Reserve. In Ramakrishnan, P.S., Purohit, A.N., Saxena, K.G., Rao, K.S. and Maikhuri, R.K. (eds.), Conservation and Management of Biological Resources in Himalaya. New Delhi: Oxford and IBH Publishing Company Private Limited, 521-545 pp.

55. Samant, S.S., Dhar, U. and Rawal, R.S. (1998a): Biodiversity status of a protected area of west Himalaya. 1Askot Wildlife Sanctuary. International Journal of Sustainable Development and World Ecology, 5: $194-203$.

56. Samant, S.S., Dhar, U. and Palni, L.M.S. (1998b): Medicinal Plants of Indian Himalaya: Diversity Distribution Potential Values. Nainital: Gyanodaya Prakashan, pp. 163.

57. Samant, S.S., Joshi, H.C. and Arya, S.C. (2003): Planning for the conservation and management of natural resources using participatory approach. A case study from Pindari area of Nanda Devi Biosphere Reserve, west Himalaya, India. Van Vigyan, 38 (1-4): 55-81.

58. Samant, S.S., Joshi, H.C. and Arya, S.C. (2000): Diversity, nativity and endemism of vascular plants in Pindari area of Nanda Devi Biosphere Reserve- II. Himalayan Biosphere Reserves, 2 (1\&2): 1-29.

59. Samant, S.S., Dhar, U. \& Rawal, R.S. (2001): Diversity, rarity and economic importance of wild edible plants of west Himalaya. Indian Journal of Forestry, 24 (2): 256-264.

60. Samant, S.S., Joshi, H.C. and Arya, S.C. (2002): Studies on the structure, composition and changes of vegetation in Nanda Devi Biosphere Reserve, west Himalaya. In Sharma, J.K., Easa, P.S., Mohanan, C., Saudharan, N., and Rai, R.K. (eds.), Biosphere Reserves in India and their Management. New Delhi: Kerala Forest Research Institute and Ministry of Environment and Forests, 133-139 pp.

61. Shannon, C.E. and Wiener, W. (1963): The Mathematical Theory of Communication. Urbana: University of Illinois Press.

62. Simpson, E.H. (1949): Measurement of diversity. Nature, 163-688.

63. Singh, S.P. (1991): Structure and function of the low and high altitude grazing ecosystems and impact of the livestock component in the central Himalaya. Final Technical Report. Ministry of Environment and Forests, Government of India, New Delhi.

64. Singh, S.P., Rikhari, H.C. and Negi, G.C.S. (1995): Community patterns in an alpine meadow of Indian central Himalaya. Journal of Indian Botanical Society, 74: 529-538.

65. Sundriyal, R.C. (1989): Assessment of grazing ability of an alpine pasture in the Garhwal Himalaya, India. Environment and Ecology, 7(1): 247-249.

66. Sundriyal, R.C. (1992): Structure, productivity and energy flow in a alpine grassland community. Journal of Vegetation Science, 3: 15-20.

67. Sundriyal, R.C., Joshi, A.P., and Gupta, S.K. (1988): Effect of free grazing on population distribution of primary producers compartment in a alpine ecosystem. Bangladesh Journal of Botany, 17: 13-18.

68. Sundriyal, R.C., Chauhan, B.B., Kandwal, S.K., and Joshi, A.P. (1987): Vegetation composition of certain grasslands of Garhwal Himalaya as determined by soil profile and seasonal variations. Indian Journal of Ecology, 14(1): 37-46.

69. Uniyal, S.K., Awasthi, A. and Rawat, G.S. (2002): Current status and distribution of commercially exploited medicinal and aromatic plants in upper Gori Valley, Kumaun Himalaya, Uttaranchal. Current Science, 82(10): 1246-1252. 